\title{
Phenotypic and Genotypic Characterization of Multi-Drug Resistant Mycobacterium tuberculosis
}

\author{
${ }^{1}$ Aliyu, M. S., , ${ }^{2}$ Garba, I., ${ }^{1}$ Tijjani, M. B., , $D o k o$, M. H .I., ${ }^{3}$ Mukhtar G. L., ${ }^{1}$ Musa, B. and \\ ${ }^{1}$ Madika, A. \\ ${ }^{1}$ Department of Microbiology, Faculty of Life Sciences, Ahmadu Bello University, Zaria \\ ${ }^{2}$ Department of Medical Microbiology, Faculty of Medical Laboratory Science, Usmanu Danfodiyo \\ University, Sokoto \\ ${ }^{3}$ Department of Microbiology, Faculty of Natural and Applied Sciences, Umaru Musa Yar'adua \\ University, Katsina
}

\begin{abstract}
This study characterized multi-drug resistant M. tuberculosis phenotypically by LJ-proportion method and genotypically by Geno Type MTBDR plus LPA. Out of the forty M. tuberculosis isolates tested, two (5.0\%) were found to be multi-drug resistant by $L J$ proportion method and one $(2.5 \%)$ was MDR by LPA. None was found to be mono-resistant to any of the drugs by LJ however, one isolate was mono resistant to RIF and one was mono resistant to INH by LPA. Comparison of Geno Type MTBDRplus LPA and phenotypic LJ-proportion methods showed that one isolate was mono resistant to RIF and one was mono resistant to INH by LPA, one and two $M D R-T B$ isolates respectively were characterized by genotypic and phenotypic methods. The remaining isolates were found to be pan susceptible by both methods. One isolate was characterized as MDR with bands at rpoB MUT2A region and ihnA MUT2 corresponding to H526Y and $A 16 G$ mutations respectively. Rifampicin mono resistance with band at rpoB MUT3 corresponding to $5531 \mathrm{~L}$ was found in one isolate. Also, isoniazid mono resistance was observed in one isolate with ihnA MUT2 band corresponding to A16G mutation. This study has shown an overall high prevalence of MDR-TB in the study area which needs to be urgently addressed. Laboratory facilities for rapid drug resistance detection are needed across the country for early and accurate diagnosis of TB and drug resistant cases. This remains an important step in managing TB drug resistance in Nigeria.

Key words: Multi-drug resistance, M. tuberculosis, phenotypic, genotypic, LJ-proportion, Geno Type MTBDRplus LPA, RIF, INH
\end{abstract}

\section{INTRODUCTION}

Multidrug-resistant (MDR) strains of Mycobacterium tuberculosis have emerged worldwide. In many countries and regions, these resistant strains constitute a serious threat to the efficacy of tuberculosis control programs. An important element in gaining control of this epidemic is developing an understanding of the molecular basis of resistance to the most important antituberculosis drugs; isoniazid and rifampicin. On the basis of this information, more exacting laboratory testing, and ultimately more appropriate and timely treatment regimens, can be developed (Somoskovi et al., 2001).

Drug resistance in $M$. tuberculosis occurs as a result of random spontaneous chromosomal mutations during natural cell replication. These mutations are not drug induced and are not linked. The probability of a drug-resistant mutant occurring is directly proportional to the size of the bacterial population. The frequency of primary resistant organisms varies for each drug; however, it is usually 1 in $10^{6}$ to 1 in $10^{8}$ (Iseman, 1993).

Spontaneous resistance to isoniazid is estimated to occur once in every $10^{6}$ organisms, and to rifampicin once in every $10^{8}$ organisms. The probability of spontaneous mutants being simultaneously resistant to two or more drugs is the product of the individual mutants. The development of drug resistance is a man made amplification of a naturally occurring phenomenon. Previous treatment for tuberculosis predisposes to the selection of multi drug resistant organisms. Non compliance is a major factor in allowing the resistant organisms to survive (Iseman, 1993). Multi drug therapy is used to prevent the emergence of drug resistant mutants during the long duration of treatment.

Resistance can be classified as single-drug, multi-drug, or poly-drug resistance depending 


\section{UJMR, Volume 5 Number 1, June, 2020, pp 24 - $30 \quad$ ISSN: 2616-0668}

on the number of drugs and/or which drugs are involved (Rieder, 1999).

Although an unequal global distribution of drug resistance exists between poor and rich countries, the problem is global. The regions where drug-resistant TB is more prevalent lack the resources to implement adequate measures to control even the susceptible types of the disease (Espinal et al., 2001; Cohen et al., 2003).

Molecular methods for MDR-TB detect the common mutations conferring resistance to RIF and $\mathrm{INH}$, rather than the resistance phenotype. The commercially available line probe assays involve DNA extraction, polymerase chain reaction (PCR), and solid phase reverse hybridization of amplified DNA to probes covering the core region of the target gene, immobilized on a nitrocellulose strip. These tests can be applied on MTB isolates or on smear positive sputum (De Beenhouwer et al., 1995; Rossau et al., 1997).

The GenoType ${ }^{\circledR}$ MTBDR assay (Hain Life sciences, Nehren, Germany) simultaneously detects the common mutations in the rpoB and katG gene (Hillemann et al., 2005). The GenoType $\circledast$ MTBDRplus, a newer version of the genotype MTBDR detects more of the common mutations in the rpoB and kat $G$ genes, and also mutations in the inhA promoter region, making it the most sensitive line probe assay for detection of resistance (Hillemann et al., 2007).

Evaluation studies of these assays have reported sensitivity and specificity of $98-100 \%$ for rifampicin, and of $70-100 \%$ for isoniazid, with results in 1-3 days (Ling et al., 2008).

Most of these studies were performed in developed countries and there is limited data on the performance of the tests in developing countries (Ling et al., 2008). A major limitation of these assays in developing countries could be the expertise in molecular biology required to perform them correctly, the unidirectional work flow laboratory infrastructure and the cost of molecular assays.

This study therefore, was aimed at characterizing multi-drug resistant $M$. tuberculosis by phenotypic and genotypic techniques in Zaria, Kaduna State.

\section{MATERIALS AND METHODS \\ Mycobacterial Isolates}

The test isolates $M$. tuberculosis were obtained from National Tuberculosis and Leprosy Training Centre, Saye, Zaria, Nigeria. The isolates were confirmed by standard Microbiological techniques. They were examined for morphology by making a smear UMYU Journal of Microbiology Research and staining by the Ziehl-Neelsen procedure. Typical acid fast bacilli were further subjected to test according to the manufacturer's instructions by SD BIOLINE TB Ag MPT64 Rapid; a rapid immuno chromatographic identification test for the M. tuberculosis complex (MTBC) that uses mouse monoclonal anti-MPT64. The kit has sensitivity and specificity of $98.6 \%$ and $100 \%$ respectively. The test cassette consists of a sample pad, a gold conjugate pad, a nitrocellulose membrane and an absorbent pad. Mouse monoclonal anti-MPT64 was immobilized on the nitrocellulose membrane as the capture material (test line). Another antibody which recognized another epitope of MPT64, conjugated with colloidal gold particles was used for antigen capture and detection in a sandwich type assay.

Four colonies were suspended in $200 \mu \mathrm{l}$ of the extraction buffer prior to the test. The cassette was removed from the foil pouch and placed on a flat dry surface. One hundred micro litres $(100 \mu \mathrm{l})$ of the suspended colonies in buffer was added into the sample well. As the test began to run, a purple colour moved across the result window in the centre of the device. After 15 minutes of sample application, the appearance of two colour bands ("T" test band and " $C$ " control band) within the result window was considered a positive result. Confirmed MTBC isolates were stored at $-20^{\circ} \mathrm{C}$ for further use.

Drug susceptibility assays

Susceptibility to isoniazid (INH) and rifampicin (RIF) was determined by the proportion method on Lowenstein Jensen egg based slopes containing different concentrations of $\mathrm{INH}$ and RIF $(0.2 \mu \mathrm{g} / \mathrm{ml}$ and $40 \mu \mathrm{g} / \mathrm{ml}$ respectively) (Canetti et al., 1969). Standard antibiotic powders (INH and RIF) were obtained from Sigma-Aldrich (Lot. No. SLBC 3024V and SLBD respectively).

The inoculum was prepared by directly suspending colonies grown for approximately three weeks on Lowenstein Jensen drug free slopes to a turbidity equivalent to a 1.0 MacFarland standard. The 1.0 MacFarland standardized suspension was further diluted to $10^{-1}, 10^{-2}$ and $10^{-3}$. The $10^{-1}$ suspension was subsequently inoculated on the drug-containing medium. Three drug-free LJ slopes were inoculated with 1:10, 1:100 and 1:1000 diluted suspensions of a 1.0 MacFarland standardized inoculum. This was done for each sample tested. The drug-susceptible MTB reference strain ATCC 27294 (H37Rv) was used as a susceptible control and known resistant strains (ATCC $35825 \mathrm{H} 37 \mathrm{Rv}$ for INH and ATCC35838 


\section{UJMR, Volume 5 Number 1, June, 2020, pp 24 - $30 \quad$ ISSN: 2616-0668}

H37Rv for RIF) were used as resistant controls. The slopes were incubated at $37^{\circ} \mathrm{C}$ and read after 4 and 6 weeks. An isolate was considered Genotypic assays were carried out by the GenoType ${ }^{\circledR}$ MTBDRplus molecular line probe assay according to the manufacturer's specifications as follows:

\section{DNA extraction}

Sterile plastic loops (one per sample) were used to suspend colonies from the LJ slants into $2 \mathrm{ml}$ micro centrifuge tubes containing $100 \mu \mathrm{l}$ molecular grade water and centrifuged at $10,000 \times$ g for $15 \mathrm{~min}$. The supernatant was discarded and the pellet re-suspended in molecular grade water, to ensure that the suspension of extracted DNA was free of impurities that might inhibit the PCR. The resuspended solutions were vortexed until they appeared slightly opaque (milky). The tubes were arranged in floating racks, placed in water bath and heated at $95^{\circ} \mathrm{C}$ for $20 \mathrm{~min}$. This was to kill the bacilli and partially lyse the cells, thereby rendering the solution non infectious and to obtain a higher yield of DNA. The tubes were placed in ultrasonic bath and incubated for $15 \mathrm{~min}$. The powerful ultrasonic shockwaves created by the sonicator disrupted the cell walls of the tubercle bacilli, causing further cell lyses, and releasing the DNA and other cell debris into the molecular grade water. Finally, the tubes were centrifuged at maximum speed $(10,000 \times \mathrm{xg})$ to separate the impure cell debris (containing the cell wall, proteins and other macromolecules) from the DNA. The heavier debris formed the pellet and the lighter DNA (free from impurities) was suspended in the supernatant. The supernatants were transferred into clean labeled micro centrifuge tubes for further use. PCR amplification of the extracted DNA

The master mix was prepared in clean DNA-free room. The master mix was made of five components with a total volume of $45 \mu \mathrm{l}$ for each PCR reaction $(35 \mu l$ of the primer nucleotide mix (PNM), $5 \mu \mathrm{l}$ of buffer, $2 \mu \mathrm{l}$ of $\mathrm{MgCl}_{2}, 3 \mu \mathrm{l}$ of molecular grade $\mathrm{H}_{2} \mathrm{O}$ and $0.2 \mu \mathrm{l}$ of Taq polymerase). The master mix was then well mixed and $45 \mu$ required for each specimen was transferred into the PCR tubes and $5 \mu \mathrm{l}$ of each sample was added to the corresponding tube containing $45 \mu \mathrm{l}$ of master mix and then mixed by gently pipetting up and down a few times. The tubes were mixed slightly and spun down for 5 - 10 seconds in a mini-centrifuge before they were placed into the thermal cycler for amplification. A 30 cycle $(10+20)$ thermal cycler program was used for the amplification. This involved ten cycles of denaturation at 95 ${ }^{\circ} \mathrm{C}$ for 30 seconds and elongation at $58{ }^{\circ} \mathrm{C}$ for resistant if the proportion of bacilli resistant to the critical concentration of a drug exceeded 1\% (Canetti et al., 1963; Canetti et al., 1969). 120 seconds; followed by an additional 20 cycles of denaturation at $95{ }^{\circ} \mathrm{C}$ for 25 seconds, annealing at $53^{\circ} \mathrm{C}$ for 40 seconds, elongation at $70^{\circ} \mathrm{C}$ for 40 seconds and final extension at $70{ }^{\circ} \mathrm{C}$ for 8 minutes. The amplicons were used for further analysis.

\section{Denaturation of DNA}

The TwinCubator ${ }^{\circledR}$ (shaking water bath) was pre-warmed to $45{ }^{\circ} \mathrm{C}$ and $20 \mu$ of denaturation solution $(\mathrm{NaOH})$ was added to each labeled well of the TwinCubator ${ }^{\circledR}$ tray followed by the addition of $20 \mu$ l of the amplicons respectively. The mixture was mixed gently by pipetting up and down five times and then incubated at room temperature for $5 \mathrm{mins}$.

\section{Hybridization and detection}

One (1) $\mathrm{ml}$ of the pre-warmed hybridization buffer (HYB) was carefully added to the wells using a pipette. The tray was then carefully tilted back and forth so that the purple denaturation solution and green hybridization buffer were thoroughly mixed. The tray was placed on the TwinCubator ${ }^{8}$ and the labeled strips added to each well ensuring that the strips were completely covered by the liquid. This was incubated at $45^{\circ} \mathrm{C}$ for 30 mins. After incubation, the glass panel lid was opened and the condensate that formed during the incubation wiped off. The HYB buffer was aspirated completely from each well using a Pasteur pipette. One (1) $\mathrm{ml}$ of the pre-warmed red stringent wash buffer (STR) was dispensed into the tray using a multi-channel pipette avoiding the contact of the strips with the tips. After 15 minute incubation at $45{ }^{\circ} \mathrm{C}$ in the TwinCubator ${ }^{\circledR}$, STR buffer was aspirated and disposed of with a Pasteur pipette. The STR buffer was washed off with $1 \mathrm{ml}$ of Rinse solution (RIN) for 1 minute. One (1) $\mathrm{ml}$ of the Conjugate (CON) solution was dispensed into each well and the glass panel lid was closed and incubated for 30 minutes on the TwinCubator®. The strips were washed twice with $1 \mathrm{ml}$ of Rinse solution (RIN) for 1 minute in the TwinCubator ${ }^{\circledR}$ to wash off the excess CON solution. One (1) $\mathrm{ml}$ of sterile distilled water was added to each strip-containing well, and a 1 minute wash performed on the TwinCubator ${ }^{\circledR}$ to wash off the RIN solution after which the distilled water was completely decanted. One (1) $\mathrm{ml}$ of the Substrate solution was dispensed into each well and incubated for 15 minutes on the TwinCubator ${ }^{\circledR}$ after which the Substrate solution was aspirated and the strips washed twice with sterile distilled water. A pair of clean tweezers was used to remove the strips 
from the TwinCubator ${ }^{\circledR}$ tray and placed onto absorbent paper. The developed strips were partially dried and transferred to the GenoType ${ }^{\circledR} \quad$ MTBDRplus score sheet.
RESULTS

Out of the forty M. tuberculosis isolates tested, two $(5.0 \%)$ were found to be multi-drug resistant by LJ-proportion method and one (2.5\%) was MDR by LPA. None was found to be
26

mono-resistant to any of the drugs by LJproportion method, however, one isolate was mono resistant to RIF and one was mono resistant to INH by LPA (Figure I).

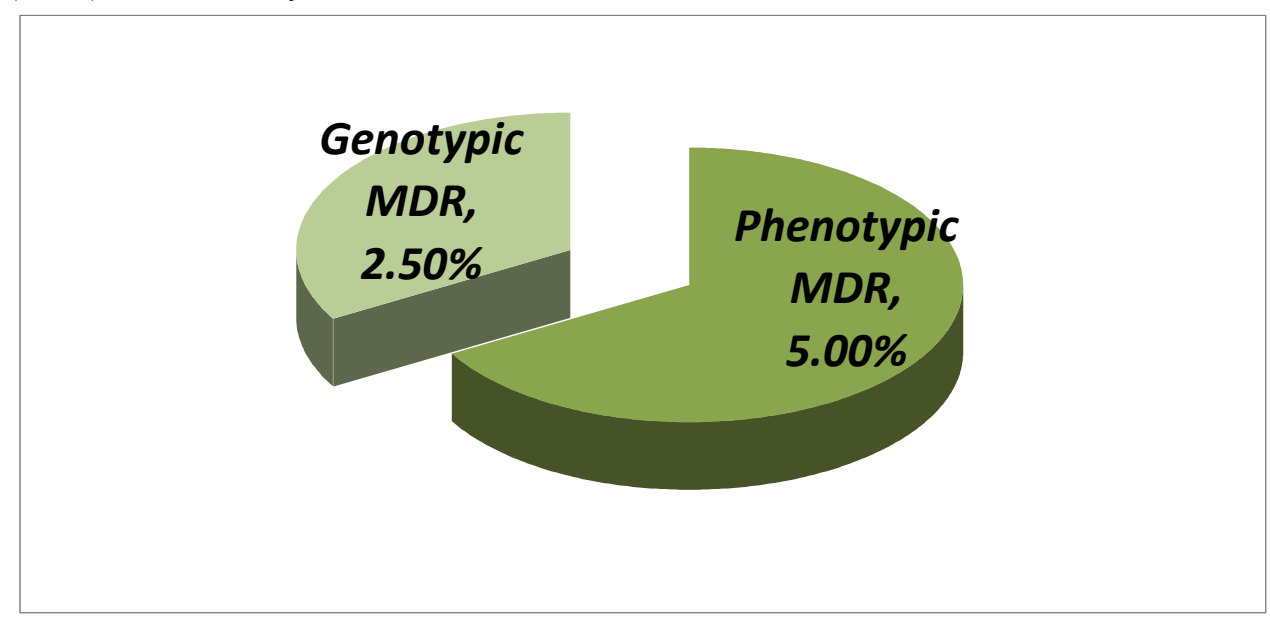

Figure I: Phenotypic and Genotypic Multi drug resistant M. tuberculosis

Table 1 shows the comparison of Geno Type MTBDRplus LPA and phenotypic LJ-proportion methods. The comparison showed that one isolate was mono resistant to RIF and one was mono resistant to INH by LPA only and none of such by the LJ Proportion method, one and two MDR-TB isolates respectively were characterized by genotypic and phenotypic methods. The remaining isolates were found to be pan susceptible by both methods.

Table 1: Comparison of Geno Type MTBDRplus LPA and phenotypic LJ-proportion methods

\begin{tabular}{lll}
\hline Susceptibility & Geno Type MTBDRplus & phenotypic LJ-proportion \\
\hline & 1 & 0 \\
RIF mono-resistance & 1 & 0 \\
INH mono-resistance & 1 & 2 \\
MDR-TB & 37 & 38 \\
Pan susceptible & 40 & 40 \\
Total & & \\
\hline
\end{tabular}

The banding patterns of mutations associated with rifampicin and isoniazid resistance in multidrug resistant and mono-resistant strains detected by MTBDR plus is shown in Table 2 . One isolate was characterized as MDR with bands at rpoB MUT2A region and ihnA MUT2 corresponding to H526Yand A16G mutations respectively. Rifampicin mono resistance with band at rpoB MUT3 corresponding to S531L was found in one isolate. Also, isoniazid mono resistance was observed in one isolate with ihnA MUT2 band corresponding to A16G mutation. 
UJMR, Volume 5 Number 1, June, 2020, pp 24 - $30 \quad$ ISSN: 2616-0668

Table 2: Patterns of gene mutations detected by Geno Type MTBDRplus assay in drug resistant M. tuberculosis isolates

\begin{tabular}{|c|c|c|c|c|c|}
\hline Gene & Band & $\begin{array}{l}\text { Gene region or } \\
\text { mutation }\end{array}$ & $\begin{array}{l}\text { MDR strains(n } \\
=1)\end{array}$ & $\begin{array}{l}\text { RIF mono resistant } \\
\text { strains }(n=1)\end{array}$ & $\begin{array}{l}\text { INH mono resistant } \\
\text { strains }(n=1)\end{array}$ \\
\hline \multicolumn{6}{|l|}{$r p o B$} \\
\hline & WT1 & 506-509 & & & \\
\hline & WT2 & 510-513 & & & \\
\hline & WT3 & 513-517 & & & \\
\hline & WT4 & 516-519 & & & \\
\hline & WT5 & $518-522$ & & & \\
\hline & WT6 & $521-525$ & & & \\
\hline & WT7 & $526-529$ & & & \\
\hline & WT8 & $530-533$ & & & \\
\hline & MUT1 & D516V & & & \\
\hline & MUT2A & H526Y & 1 & & \\
\hline & MUT2B & H526D & & & \\
\hline & MUT3 & S531L & & 1 & \\
\hline \multicolumn{6}{|l|}{ katG } \\
\hline & WT & 315 & & & \\
\hline & MUT1 & S315T1 & & & \\
\hline & MUT2 & S315T2 & & & \\
\hline \multicolumn{6}{|l|}{ inhA } \\
\hline & WT1 & $-15 /-16$ & & & \\
\hline & WT2 & & & & \\
\hline & MUT1 & C15T & & & \\
\hline & MUT2 & A16G & 1 & & 1 \\
\hline & MUT3A & T8C & & & \\
\hline & MUT3B & T8A & & & \\
\hline
\end{tabular}

Key: WT = Wild Type, MUT $=$ Mutant, $\mathrm{D}=$ Aspatate, $\mathrm{V}=$ Valine, $\mathrm{H}=$ Histadine, $\mathrm{Y}=$ Tyrosine, $\mathrm{S}=$ Serine, $\mathrm{L}=$ Leucine, $\mathrm{T}=$ Threonine, $\mathrm{C}=$ Cysteine, $\mathrm{A}=$ Alanine, $\mathrm{G}=\mathrm{Glycine}$

DISCUSSION

The most important measure of TB drug resistance is the number of new cases that are MDR-TB (Dye et al., 2002). This study showed a prevalence rate of $5.0 \%(2 / 40)$. The development of drug resistance is a man made amplification of a naturally occurring phenomenon. Previous treatment for tuberculosis predisposes to the selection of multi drug resistant organisms. Non compliance is a major factor in allowing the resistant organisms to survive. The availability of drugs in the open market and a private sector that delivers drugs to the population in an unregulated fashion in Nigeria could also be factors that might favour development of MDRTB.

The genotypic drug resistance assay revealed that all the resistant isolates were heteroresistant; a phenomenon where some cells within a population may remain susceptible to the antibiotic, whereas other cells display varying degrees of drug resistance. This was determined by the simultaneous detection of the wild type and mutant molecular susceptibility (Foundation for innovative new diagnosis, 2012).
In patients infected with a fully susceptible strain, drug resistance can develop gradually during inadequate treatment due to selection of cells with random mutations in sites associated with drug resistance (i.e. secondary resistance). In this case, as the proportion of susceptible cells decrease and resistant cells increase, a hetero-resistant population of cells will be present. These cells are primarily identical throughout the genome but a proportion of the population differs in sites associated with drug resistance (Foundation for innovative new diagnosis, 2012).

In contrast, patients infected with fully susceptible strains may develop mixed infections if they are co-infected with another strain that is drug resistant, resulting in a mixed population of two genetically distinct strains, one drug susceptible, and the other drug resistant.

Also, hetero-resistance likely represents a natural variation in the population of cells of M. tuberculosis (Foundation for innovative new diagnosis, 2012). Prescription of inadequate treatment regimen, irregular drug supply, poor drug quality with low bioavailability, and poor compliance among the study population could 
be attributed to the development of heteroDiscordance between genotypic and phenotypic assays was observed in one isolate with RIF mono resistance. The isolate was classified as MDR by the phenotypic assay. This could be due to silent mutation or synonymous single nucleotide polymorphism (sSNP) at the target site. Silent mutations do not result in structural changes in the inhA and so do not interfere with its inhibition by INH. Moreover, findings of silent mutations in inhA are not surprising as SNPs occur every $3 \mathrm{~kb}$ of MTB genome (Comas et al., 2011). Ando, et al. (2014) reported a silent mutation in a significant number of $\mathbf{I N H}$ resistant $M$. tuberculosis clinical isolates. Mutations conferring INH resistance in other genes such as mabA (G609A) (Ando et al., 2014) aphC (alkyl hydroperoxide reductase), kasA ( $\mathrm{B}$ ketoacyl-ACP synthase) and nadh (NADH dehydrogenase) (Cohn et al., 1997; Balasubramanian et al., 2012) not included in Geno Type MTBDRplus have also been reported. The critical concentration of INH on LJ medium which is being used for over 50 years is $0.2 \mu \mathrm{g} / \mathrm{ml}$ (Jamieson et al., 2014). Lower critical concentrations of $0.0312 \mu \mathrm{g} / \mathrm{ml}$ for low-

\section{REFERENCES}

Ando, H., Miyoshi-Akiyama. T., Watanabe, S. and Kirikae, T. (2014). A silent mutation in mabA confers isoniazid resistance on Mycobacterium tuberculosis. Molecular Microbiology, 91(3):538-547.

Balasubramanian, S., Gayathri, R., Madhavan, H.N., Natrajan, S. and Lily Therese, K. (2012). Phenotypic and genotypic characteristics of drug resistance in Mycobacterium tuberculosis isolates from pediatric population of Chennai, India. Indian Journal of Medical Microbiology, 30(4):411-417

Canetti, G., Froman, S., Grosset, J., Hauduroy, P., Langerova, M., Mahler, H.T., Meissner, G., Mitchison, D.A., and Sula, L. (1963). Mycobacteria: Laboratory methods for testing drug sensitivity and resistance. Bulletin of the World Health Organization, 29:565-578.

Canetti, G., Fox, W., Khomenko, A., Mahler, H.T., Menon, N.K., Mitchison, D.A., Rist, N., Smelev, N.A. (1969). Advances in techniques of testing mycobacterial drug sensitivity, and the use of sensitivity tests in tuberculosis control program. Bulletin of World Health Organization, 41(1):21-43. resistance observed in this study. level resistance and $0.125 \mu \mathrm{g} / \mathrm{ml}$ for high-level resistance were reported by Gumbo, (2010).

\section{CONCLUSION AND RECOMMENDATION}

The findings showed that one isolate each was mono resistant to RIF and INH by LPA, one and two MDR-TB isolates respectively were characterized by genotypic and phenotypic methods. The remaining isolates were found to be pan susceptible by both methods. One isolate was characterized as MDR with bands at rpoB MUT2A region and innA MUT2 corresponding to H526Yand A16G mutations respectively. Rifampicin mono resistance with band at rpoB MUT3 corresponding to S531L was found in one isolate. Also, isoniazid mono resistance was observed in one isolate with innA MUT2 band corresponding to A16G mutation.

The use of robust molecular techniques such as DNA sequencing employed for the detection of occult cases with low-level resistance and other resistance not included in the Geno Type MTBDRplus kit is recommended.

Cohen, T., Sommers, B. and Murray, M. (2003). The effect of drug resistance on the fitness of Mycobacterium tuberculosis. Lancet, 3 : 13-21

Cohn, D.L., Flavia, B. And Raviglione, M.C. (1997). Drug-resistant tuberculosis: review of the worldwide situation and the WHO/IUATLD global surveillance project. Clinical Infectious Diseases, 24:S121-30.

Comas, I., Borrell, S., Roetzer, A., Rose, G., Malla, B., Kato-Maeda, M., Galagan J, Niemann S, and Gagneux, S. (2011). Whole-genome sequencing of rifampicin-resistant Mycobacterium tuberculosis strains identifies compensatory mutations in RNA polymerase genes. Nature Genetics, 44(1):106-110.

De Beenhouwer, H., Lhiang, Z., Jannes, G., Mijs, W., Machtelinckx, L., Rossau, R., Traore, H., and Portaels, F. (1995) Rapid detection of rifampicin resistance in sputum and biopsy specimens from tuberculosis patients by PCR and line probe assay. Tuberculosis and Lung Diseases, 76(5):425-430.

Dye, C., Williams, B.G., Espinal, M.A., and Raviglione, M.C. (2002). "Erasing the world's slow stain: strategies to beat 
multi drug resistant tuberculosis," Science, 295(5562): 2042-2046.

Espinal, M. A., Laszlo, A. and Simonsen, L. (2001). Global trends in the resistance to antituberculosis drugs. New England Journal of Medicine, 344:1294-1303.

Foundation for innovative new diagnosis (FIND) (2012). Molecular Detection of DrugResistant Tuberculosis by Line Probe Assay; Laboratory Manual for ResourceLimited Settings. www. finddiagnostics.org.

Gumbo, T. (2010). New Susceptibility Breakpoints for First-Line Antituberculosis Drugs Based on Antimicrobial

Pharmacokinetic/Pharmacodynamic Science and Population Pharmacokinetic Variability. Antimicrobial Agents andChemotherapy, 54 (4):1484-1491.

Hillemann, D., Weizenegger, M., Kubica, T., Richter, E., and Niemann, S. (2005). Use of the Geno Type MTBDR assay for rapid detection of rifampin and isoniazid resistance in Mycobacterium tuberculosis complex isolates. Journal of Clinical Microbiology,43 (8):36993703.

Hillemann, D., Rusch-Gerdes, S., and Richter, E. (2007). Evaluation of the GenoType MTBDRplus assay for rifampin and isoniazid susceptibility testing of Mycobacterium tuberculosis strains and clinical specimens. Journal of Clinical Microbiology, 45 (8):2635-2640.

Iseman, M. D. (1993). Treatment of multidrug resistant tuberculosis. New
England Journal of Medicine; 329: 784791

Jamieson, F.B., Guthrie, J.L., Neemuchwala, A., Lastovetska, O., Melano, R.G., and Mehaffy, C.(2014). Profiling of rpoB mutations and MICs for rifampin and rifabutin in Mycobacterium tuberculosis. Journal of Clinical Microbiology, 52 (6):2157-2162.

Ling, D.I., Zwerling, A.A., and Pai, M. (2008). GenoType MTBDR assays for the diagnosis of multidrug-resistant tuberculosis: a meta-analysis. European Respiratory Journal, 32(5):1165-1174. Mycobacterium tuberculosis. Lancet, 3:13-21

Rieder, H.L. (1999). Epidemiologic basis of tuberculosis control. $1^{\text {st }}$ edition: International Union Against Tuberculosis and Lung Disease, Paris. Pp. 162.

Rossau, R., Traore, H., De Beenhouwer, H., Mijs, W., Jannes, G., De Rijk, P. and Portaels, F. (1997). Evaluation of the INNO-LiPA Rif. TB assay, a reverse hybridization assay for the simultaneous detection of Mycobacterium tuberculosis complex and its resistance to rifampin. Antimicrobial Agents and Chemotherapy, 41(10):2093-2098.

Somoskovi, A., Parsons, M. L. and Salfinger, M. (2001): The molecular basis of resistance to isoniazid, rifampin, and pyrazinamide in Mycobacterium tuberculosis. Respiratory Research 2(3). 164-169 